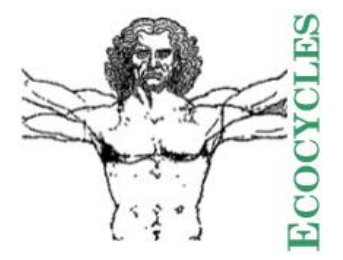

Ecocycles, Vol. 6, No. 2, pp. 19-24 (2020)

DOI: $10.19040 /$ ecocycles.v6i2.173

\title{
OPINION
}

\section{Human geography of drylands. I. Planning the database: Physical, built-up, chemical, biological (ecological), and social indicators}

\author{
Matyas Arvai ${ }^{1}$, Karoly Fekete ${ }^{2}$, Laszlo Pasztor ${ }^{1}$, Tamas Komives ${ }^{3,4}$ \\ ${ }^{1}$ Institute for Soil Sciences and Agricultural Chemistry, Centre for Agricultural Research, LERN, Herman Otto 15, 1022 Budapest, Hungary; \\ ${ }^{2}$ Lake Balaton Development Coordination Agency, Batthyany u. 1, 8600 Siófok, Hungary; Karoly Robert Campus, Szent Istvan University, \\ Matrai ut 36, 3200 Gyongyos, Hungary, ${ }^{4}$ Plant Protection Institute, Centre for Agricultural Research, LERN, Herman Otto 15, 1022 \\ Budapest, Hungary
}

E-mail addresses: arvai.matyas@atk.hu, karoly.fekete@,balatonregion.hu, pasztor.laszlo@atk.hu, komives.tamas@atk.hu

\begin{abstract}
We propose a method based on multilayered mapping for investigating the current problems of people who live in drylands and we urge decision-makers to support such studies to establish the foundations for future decisive and preventive actions. This paper contains an expandable compilation of the environmental indicators (mostly mappable) that may influence the human geography of a certain region. We believe that this geospatial approach may help to resolve convoluted physical, chemical, and social relationships and, at the same time, generate a valuable database for further research. The application of the concept, if successful, will give directions to tackle certain contemporary problems in drylands and predict future ones caused by global climate change.
\end{abstract}

Keywords - drylands, human geography, database, GIS, geoinformation, geodata, multilayered mapping, social sciences

\section{...the least initial deviation from the truth is multiplied later a thousandfold.}

Aristotle, $350 \mathrm{BC}$

\section{INTRODUCTION}

The rapid development of the geographic information system (GIS) during the last quarter of a century has led to a diversity of uses in data visualization and analysis and paved the method's way for a wide range of scientific disciplines, including agriculture (Gaborjanyi et al., 2003), geology, environmental science (Healy and Walshe, 2019), and most recently in social sciences (Ballas et al., 2017; Carter 2019; Lechner et al., 2019). Thus, human geography (also called anthropogeography) studies investigate political-economic, cultural-social, and human-environment relations by using the multilayered mapping feature of GIS to resolve convoluted social relationships and, at the same time, generate valuable databases for further research ( $\mathrm{Li}$ et al., 2019). The most important benefit of the method is that it can visualize a vast variety of data. From the maps, valuable information can be extracted and used in feasibility studies or to achieve better-informed decisions (Ballas et al., 2017).

In this paper, we outline a concept of a) using multilayered mapping for studying current, day-to-day existential problems of people who live in drylands and b) present an expandable compilation of the environmental indicators (mostly mappable) that may influence the human geography of a dryland region. The application of the concept, if successful, will give directions to tackle certain contemporary problems and predict future ones caused by global climate change in drylands.

\section{DRYLANDS}

Approximately one-fifth of the total surface area of the Earth is defined as habitable by humans (Cervigni and Morris, 2016). Human habitats are conditions in which people live. Besides, to be accessible, a human habitat needs to be able to 
provide shelter, uncontaminated water, clean energy, unpolluted environment, and an adequate amount of nutritious food. Furthermore, the habitat should not be vulnerable to climate or other natural or human hazards (Filho et al., 2018).

Dryland type human habitats are specific areas defined by a scarcity of water. The United Nations Environment Program defines drylands as tropical and temperate areas characterized with an aridity index (AI, the ratio of the annual precipitation and potential evapotranspiration totals) less than 0.65 (Plaza et al., 2018).

All dryland countries, although widely different in many respect (resources, opportunities, etc.), need to address continuously problems related to the variability (and possible further changes) in the climate that influence the countries' viability and may lead to social turbulence. COST Action CA16233 of the European Union has been initiated to improve the coordination of drylands research between scientific disciplines and different geographical areas. (Anon., 2019).

To carry out a human geography project the following tasks need to be executed:

a) database design: selection of indicators for multilayered mapping

b) data collection

c) data processing, analysis, and interpretation

d) proposals for tackling the current key problems of the study area and forecasting future ones

e) action, followed by monitoring and reflection.

\section{DESIGN OF THE DATABASE}

\section{Selection of indicators}

We propose, for consideration, the indicators listed in Tables 1-12 as possible attributes (preferably in time-course format) for building a geoinformation database on drylands.

Naturally, except for the general indicators in Table 1, during the construction of the database, all Tables 2 to 12 would be united into a single table of attributes. Our list of indicators in the Tables, although it covers a wide range of environmental factors (physical, chemical, biological, and social) is not intended to be complete: criticism, additions, and corrections are welcome.

\section{Indicator redundancy and identifiability}

The number of indicators included in this paper may be considered unnecessarily extensive. Besides, several of the indicators listed in the Tables do not have yet established, widely-accepted scientific definitions, and quantifiable measures and units. Pilot investigations need to address the complex problems of indicator redundancy and identifiability using well-established complex mathematical models (Little et al., 2010).

For practical reasons, a pilot study may use a very narrow set of dryland-specific indicators and a composite indicator that characterizes the human carrying capacity of the region studied.

\section{Table 1. General information on the country studied}

\begin{tabular}{|r|l|l|}
\hline & Indicator & \multicolumn{1}{|c|}{ / U * } \\
\hline 1 & Area & $\mathrm{km}^{2}$ \\
\hline 2 & Drylands area & $\mathrm{km}^{2}$ \\
\hline 3 & Water area & $\mathrm{km}^{2}$ \\
\hline 4 & Gross domestic product per capita & $\mathrm{USD}$ \\
\hline 5 & Gross national product per capita & USD \\
\hline 6 & National debt & percent GDP \\
\hline 7 & Inflation rate & percent \\
\hline 8 & Population size & number \\
\hline 9 & Human development index & normalized \\
\hline 10 & 5-year average growth of GDP & percent \\
\hline 11 & Level of urbanization & 3 categories \\
\hline 12 & Proportion of science expenditure & percent \\
\hline 13 & Proportion of education expenditure & percent \\
\hline 14 & Proportion of welfare expenditure & percent \\
\hline
\end{tabular}

* Dimension / unit

Table 2. Physical and natural environment: resources and hazards [mappable data]

\begin{tabular}{|c|c|c|}
\hline & Indicator & $\mathbf{D} / \mathbf{U} *$ \\
\hline 1 & Average yearly temperature & ${ }^{\circ} \mathrm{C}$ \\
\hline 2 & Average monthly temperature & ${ }^{\circ} \mathrm{C}$ \\
\hline 3 & Sunshine duration & $\mathrm{h} /$ year \\
\hline 4 & Relative humidity & percent \\
\hline 5 & Precipitation (yearly) & $\mathrm{mm}$ \\
\hline 6 & Precipitation (monthly) & $\mathrm{mm}$ \\
\hline 7 & Volume of collected precipitation & $\mathrm{km}^{3}$ \\
\hline 8 & Aridity Index & composite \\
\hline 9 & Number of reservoirs & number \\
\hline 10 & Reservoir capacity & $\mathrm{km}^{3}$ \\
\hline 11 & Irrigated area & $\mathrm{km}^{2}$ \\
\hline 12 & Total water consumption & $\mathrm{km}^{3}$ \\
\hline 13 & Residential water consumption & $\mathrm{km}^{3}$ \\
\hline 14 & Disasters: crop area affected & $\mathrm{km}^{2}$ \\
\hline 15 & Disasters: size of affected population & percent \\
\hline 16 & Disasters: economic losses & per centGDP \\
\hline 17 & Flooding hazard & composite \\
\hline 18 & Land area of the admin. district & $\mathrm{km}^{2}$ \\
\hline 19 & Arable land & $\mathrm{km}^{2}$ \\
\hline 20 & Soil texture & composite \\
\hline 21 & Soil coarse fragments & composite \\
\hline 22 & Soil depth & $\mathrm{m}$ \\
\hline 23 & Soil drainage & 5 classes \\
\hline 24 & Soil available water capacity & liter $/ \mathrm{m}^{3}$ \\
\hline 25 & Soil sodicity & composite \\
\hline 26 & Soil salinity & $\mathrm{dS} / \mathrm{m}$ \\
\hline 27 & Soil pH & number \\
\hline 28 & Soil organic matter content & percent \\
\hline 29 & Soil cation exchange capacity & composite \\
\hline
\end{tabular}




\begin{tabular}{|l|l|l|}
\hline 30 & Soil calcium carbonate content & percent \\
\hline 31 & Slope & percent \\
\hline 32 & Erosion & tons/hectare \\
\hline 33 & Terrain (elevation, slope, shelter) & composite \\
\hline 34 & Seismic (earthquake) hazard & composite \\
\hline 35 & Volcanic activity hazard & composite \\
\hline 36 & Sandstorm hazard & composite \\
\hline 37 & Green areas in cities & $\mathrm{km}^{2}$ \\
\hline 38 & Crops: total area & $\mathrm{km}^{2}$ \\
\hline 39 & Forest area & $\mathrm{km}^{2}$ \\
\hline 40 & Edible wild plants & $\mathrm{Y} / \mathrm{N}$ \\
\hline 41 & Edible wild animals & $\mathrm{Y} / \mathrm{N}$ \\
\hline 42 & Predator hazard & composite \\
\hline 43 & Poisonous plant and animal hazard & composite \\
\hline 44 & Vectors of diseases & composite \\
\hline 45 & Emerging invasive species & composite \\
\hline * Dimension / unit & \\
\hline
\end{tabular}

Table 3. Built environment [mappable data]

\begin{tabular}{|r|l|l|}
\hline & Indicator & $\mathbf{D} / \mathbf{U}^{*}$ \\
\hline 1 & Built-up area & $\mathrm{km}^{2}$ \\
\hline 2 & Sewage system & percent \\
\hline 3 & Internet & Y/N \\
\hline 4 & Public lighting & Y/N \\
\hline 5 & Transport: rural access index & composite \\
\hline 6 & Transport: internatl. roughness index & composite \\
\hline 7 & Public transportation & Y/N \\
\hline 8 & Museums & number \\
\hline 9 & Theatres & number \\
\hline 1 & Film theatres & number \\
0 & & \\
\hline 11 & Concert halls & number \\
\hline 1 & Schools & number \\
2 & & \\
\hline 1 & Hospitals & number \\
3 & & \\
\hline
\end{tabular}

* Dimension / unit

Table 4. Environment: extent of pollution [mappable data]

\begin{tabular}{|l|l|l|}
\hline & Indicator & D / U * \\
\hline 1 & Pollution management projects & USD \\
\hline 2 & Environmental infrastructure projects & USD \\
\hline 3 & Utilized waste products & percent \\
\hline 4 & Waste water managed & percent \\
\hline 5 & $\begin{array}{l}\text { Industrial sulfur dioxide emission } \\
\text { managed }\end{array}$ & percent \\
\hline 6 & Industrial soot emission managed & percent \\
\hline 7 & Air pollution managed & percent \\
\hline 8 & Water pollution managed & percent \\
\hline
\end{tabular}

\section{Table 5. Social environment: population [mappable data]}

\begin{tabular}{|l|l|l|}
\hline & Indicator & $\mathbf{D} / \mathbf{U}$ * \\
\hline 1 & Population tree & image \\
\hline 2 & Average age & number \\
\hline
\end{tabular}

\begin{tabular}{|l|l|l|}
\hline 3 & Aged below 35 y & percent \\
\hline 4 & Aged below 15 y & percent \\
\hline 5 & Density & people $\mathrm{km}^{-2}$ \\
\hline 6 & Sociodynamics & composite \\
\hline 7 & Fertility rate & number \\
\hline
\end{tabular}

* Dimension / unit

Table 6. Social environment: community problems [mappable data]

\begin{tabular}{|r|l|c|}
\hline & Indicator & D / U * \\
\hline 1 & Illegal drug use & composite \\
\hline 2 & Alcohol use & composite \\
\hline 3 & Crime & composite \\
\hline 4 & Youth violence & composite \\
\hline 5 & Child abuse & composite \\
\hline 6 & Discrimination (minority issues) & composite \\
\hline 7 & Availability of recreational activities & composite \\
\hline 8 & Racism & composite \\
\hline 9 & Homelessness & composite \\
\hline 10 & Poverty & composite \\
\hline 11 & Smoking status & composite \\
\hline 12 & Obesity & composite \\
\hline 13 & Malnutrition & composite \\
\hline 14 & Housing: renters & percent \\
\hline 15 & Housing: single-family dwellings & percent \\
\hline
\end{tabular}

* Dimension / unit

\section{Table 7. Social environment: economy [mappable data]}

\begin{tabular}{|l|l|l|}
\hline & Indicator & D / U * \\
\hline 1 & Average income & USD \\
\hline 2 & Availability of food & composite \\
\hline 3 & Extractable geological materials & composite \\
\hline 4 & Tourism: guest nights & number \\
\hline 5 & Tourism: proportion of local GDP & percent \\
\hline 6 & Annual electricity consumption & $\mathrm{GW}$ \\
\hline 7 & Residential electricity consumption & $\mathrm{GW}$ \\
\hline 8 & Liquefied petroleum gas consumption & $\mathrm{m}^{3}$ \\
\hline
\end{tabular}

* Dimension / unit

\section{Table 8. Social environment: healthcare [mappable data]}

\begin{tabular}{|l|l|c|}
\hline & Indicator & $\mathbf{D} / \mathbf{U}$ * \\
\hline 1 & Health care in GDP & percent \\
\hline 2 & Life expectancy index & composite \\
\hline 3 & Mental health & composite \\
\hline 4 & Availability of hospitals & composite \\
\hline 5 & Availability of local doctors & composite \\
\hline 6 & Infectious disease hazard & composite \\
\hline
\end{tabular}

* Dimension / unit

\section{Table 9. Social environment: education [mappable data]}

\begin{tabular}{|l|l|l|}
\hline & Indicator & D / U * \\
\hline 1 & Mean years of schooling index & number \\
\hline 2 & Expected years of schooling index & composite \\
\hline 3 & Education index & composite \\
\hline
\end{tabular}




\begin{tabular}{|l|l|l|}
\hline 4 & Illiteracy & percent \\
\hline 5 & Availability of adult education & composite \\
\hline
\end{tabular}

* Dimension / unit

Table 10. Social environment: culture [mappable data]

\begin{tabular}{|r|l|c|}
\hline & Indicator & $\mathbf{D} / \mathbf{U} *$ \\
\hline 1 & Arts (performing, visual) & composite \\
\hline 2 & Education (primary, secondary, tertiary) & composite \\
\hline 3 & Literature & composite \\
\hline 4 & Gastronomy & composite \\
\hline 5 & Architecture & composite \\
\hline 6 & Politics & composite \\
\hline 7 & Clothing & composite \\
\hline 8 & Entertainment & composite \\
\hline 9 & Sports & composite \\
\hline 10 & Traditions & composite \\
\hline 11 & Mass media & composite \\
\hline 12 & Religion & composite \\
\hline
\end{tabular}

* Dimension / unit

\section{Table 11. Social environment: creativity [mappable data]}

\begin{tabular}{|c|l|c|}
\hline & Indicator & $\mathbf{D} / \mathbf{U}$ * \\
\hline 1 & Human capital index & composite \\
\hline 2 & Creative class index & composite \\
\hline 3 & Scientific talent index & composite \\
\hline 4 & Innovation index & composite \\
\hline 5 & R\&D index & composite \\
\hline 6 & Global social tolerance index & composite \\
\hline
\end{tabular}

* Dimension / unit

Table 12. Social environment: security [mappable data]

\begin{tabular}{|r|l|c|}
\hline & Indicator & D / U * \\
\hline 1 & Unemployment & percent \\
\hline 2 & Language skills & composite \\
\hline 3 & Inequality (Gini coefficient) & composite \\
\hline 4 & Work ethics & composite \\
\hline 5 & Opportunities for youth & composite \\
\hline 6 & Social structure & composite \\
\hline 7 & Social net & composite \\
\hline 8 & Social care & composite \\
\hline 9 & Democracy level & composite \\
\hline 10 & Mobility & composite \\
\hline 11 & Migration & composite \\
\hline 12 & Wealth distribution & composite \\
\hline 13 & Family structure & composite \\
\hline 14 & Leisure & composite \\
\hline 15 & Crime and public safety & composite \\
\hline
\end{tabular}

* Dimension / unit

\section{DATA COLLECTION}

Building a database of this scale is a tremendous task, that can be achieved only through a very large amount of human effort: collecting and sifting through existing quantitative and qualitative data, evaluating existing written and digital records from libraries and satellite images.
On a positive note, artificial intelligence can be used to monitor and analyze social media with automation, improved accuracy, and reduced input of human labor (Perakakis et al., 2019). Furthermore, in addition to professionals (e.g., teachers and researchers), volunteers, activists, and the general public may provide unprecedented contributions via social media and citizen science (Roy et al., 2018).

\section{DATA PROCESSING, ANALYSIS, AND INTERPRETA- TION}

Several goals may be considered when evaluating the data. For example,

a) evaluation of resource and environmental carrying capacity (Li, 2019) (Bao et al., 2020)

b) multi-criteria analysis of land suitability (Niles et al., 2015)

c) factors limiting adaptation (Nguyen et al., 2015)

d) community health, population and environment, neighborhood effects, land use, fertility, migration (Logan et al., 2010) e) population dynamics (Organ, 2019)

f) ecological risk assessment using fuzzy analytical hierarchy process (Radionovs and Užga-Rebrovs, 2016)

g) multi-criteria decision analysis (Vaissi and Sharifi, 2019) h) local and regional vulnerability assessment (Polese et al., 2020).

\section{CONCLUDING REMARKS}

This paper contains an expandable compilation of environmental indicators (mostly mappable) that may influence the human geography of a certain region. We believe that a geospatial approach may help to resolve convoluted physical, chemical, and social relationships and, at the same time, generate a valuable database for further research. The application of the concept, if successful, will give directions to tackle certain contemporary problems and predict future ones caused by global climate change.

\section{ACKNOWLEDGMENTS}

The authors gratefully acknowledge the contribution of COST Action CA 16233 and helpful discussions with Dr. Peter Pal Toth (Demography Institute, Hungarian Central Statistical Office) and Dr. Tamas Hermann (Pannon University, Keszthely).

\section{Public interest Statement}

The purpose of the paper was to outline a concept based on the application of multilayered mapping for investigating contemporary problems of inhabitants of drylands. It presents an expandable compilation of a large number of environmental indicators (mostly mappable) that may influence the human geography of a dryland territory. We conclude that the technique of multilayered mapping may help scientists and decision-makers in resolving convoluted physical, chemical, and social relationships and, at the same time, generate a valuable database for further research. Besides, the concept may provide guidance to tackle current dryland-related 
problems and forecast ones caused by global climate change.

\section{REFERENCES}

Anon., 2019. Action CA16233 [WWW Document]. COST. https://www.cost.eu/actions/CA16233/\#tabs|Name:overview (Accessed 07.17.2020).

Aristotle (350 BC), On the Heavens. Book 1, Part 5, Paragraph 1. Translated by John L. Stocks. The Internet Classics Archive.

http://classics.mit.edu/Aristotle/heavens.1.i.html (Accessed $07.20 .2020)$

Ballas, D., Clarke, G., Franklin, R.S., Newing, A., 2017. GIS and the Social Sciences: Theory and Applications. Routledge.

DOI: $\underline{10.4324 / 9781315759326}$

Bao, H., Wang, C., Han, L., Wu, S., Lou, L., Xu, B., Liu, Y., 2020. Resources and environmental pressure, carrying capacity, and governance: A case study of Yangtze River Economic Belt. Sustainability 12, 1576.

DOI: $\underline{10.3390 / \mathrm{su} 12041576}$

Carter, J.C., 2019. Introduction to Human Geography Using ArcGIS Online. Esri Press.

Cervigni, R., Morris, M., 2016. Confronting drought in Africa's drylands: Opportunities for enhancing resilience, Africa Development Forum. The World Bank.

DOI: $\underline{10.1596 / 978-1-4648-0817-3}$

Filho, W.L., Al-Amin, A.Q., Nagy, G.J., Azeiteiro, U.M., Wiesböck, L., Ayal, D.Y., Morgan, E.A., Mugabe, P., Aparicio-Effen, M., Fudjumdjum, H., Chiappetta Jabbour, C.J., 2018. A comparative analysis of climaterisk and extreme event-related impacts on well-being and health: Policy implications. Int. J. Environ. Res. Public. Health 15(2) 331.

DOI: $\underline{10.3390 / \text { ijerph15020331 }}$

Gaborjanyi, R., Pasztor, L., Papp, M., Szabo, J., Mesterhazy, Á., Nemeth, T., Komives, T., 2003. Use of remote sensing to detect virus-infected wheat plants in the field. Cereal Res. Commun. 31, 113-120.

DOI: $\underline{0.5281 / \text { zenodo. } 3054549}$

Healy, G., Walshe, N., 2019. Real-world geographers and geography students using GIS: relevance, everyday applications and the development of geographical knowledge. Int. Res. Geogr. Environ. Educ. 29, 178-196.

DOI: $\underline{10.1080 / 10382046.2019 .1661125}$

Lechner, A.M., Owen, J., Ang, M., Kemp, D., 2019. Spatially integrated social sciences with qualitative GIS to support impact assessment in mining communities. Resources 8 , 47.

DOI: $10.3390 /$ resources 8010047
Li, K., Jin, X., Ma, D., Jiang, P., 2019. Evaluation of resource and environmental carrying capacity of China's rapidurbanization areas - A case study of Xinbei District, Changzhou. Land 8, 69.

DOI: $\underline{10.3390 / \text { land } 8040069}$

Little, M.P., Heidenreich, W.F., Li, G., 2010. Indicator identifiability and redundancy: Theoretical considerations. PLOS ONE 5, e8915.

DOI: $10.1371 /$ journal.pone.0008915

Logan, J.R., Zhang, W., Xu, H., 2010. Applying spatial thinking in social science research. GeoJournal 75, 15-27.

DOI: $\underline{10.1007 / \mathrm{s} 10708-010-9343-0}$

Michalek, J., Zarnekow, N., 2012. Application of the rural development index to analysis of rural regions in Poland and Slovakia. Soc. Indic. Res. 105, 1-37.

DOI: $\underline{10.1007 / \mathrm{s} 11205-010-9765-6}$

Nguyen, T.T., Verdoodt, A., Van Y, T., Delbecque, N., Tran, T.C., Van Ranst, E., 2015. Design of a GIS and multicriteria based land evaluation procedure for sustainable land-use planning at the regional level. Agric. Ecosyst. Environ. 200, 1-11.

DOI: $10.1016 /$ j.agee.2014.10.015

Niles, M.T., Lubell, M., Brown, M., 2015. How limiting factors drive agricultural adaptation to climate change. Agric. Ecosyst. Environ. 200, 178-185.

DOI: $10.1016 /$ j.agee.2014.11.010

Organ, J., 2019. Changing weather, crop yields and Chadian population dynamics.

DOI: $\underline{10.13140 / \text { RG.2.2.19959.57768 }}$

Perakakis, E., Mastorakis, G., Kopanakis, I., 2019. Social media monitoring: An innovative intelligent approach. Designs 3, 24.

DOI: $\underline{10.3390 / \text { designs3020024 }}$

Plaza, C., Zaccone, C., Sawicka, K., Méndez, A.M., Tarquis, A., Gascó, G., Heuvelink, G.B.M., Schuur, E.A.G., Maestre, F.T., 2018. Soil resources and element stocks in drylands to face global issues. Sci. Rep. 8.

DOI: $\underline{10.1038 / \mathrm{s} 41598-018-32229-0}$

Polese, M., Di Ludovico, M., Gaetani d'Aragona, M., Prota, A., Manfredi, G., 2020. Regional vulnerability and risk assessment accounting for local building typologies. Int. J. Disaster Risk Reduct. 43, 101400.

DOI: $10.1016 /$ j.ijdrr.2019.101400

Radionovs, A., Užga-Rebrovs, O., 2016. Fuzzy analytical hierarchy process for ecological risk assessment. Inf. Technol. Manag. Sci. 19.

DOI: $10.1515 /$ itms-2016-0005

Roy, H., Groom, Q., Adriaens, T., Agnello, G., Antic, M., Archambeau, A.-S., Bacher, S., Bonn, A., Brown, P., Brundu, G., López, B., Cleary, M., Cogălniceanu, D., 
Groot, M. de, Sousa, T.D., Deidun, A., Essl, F., Pečnikar, Ž.F., Gazda, A., Gervasini, E., Glavendekic, M., Gigot, G., Jelaska, S., Jeschke, J., Kaminski, D., Karachle, P., Komives, T., Lapin, K., Lucy, F., Marchante, E., Marisavljevic, D., Marja, R., Torrijos, L.M., Martinou, A., Matosevic, D., Mifsud, C., Motiejūnaite, J., Ojaveer, H., Pasalic, N., Pekárik, L., Per, E., Pergl, J., Pesic, V., Pocock, M., Reino, L., Ries, C., Rozylowicz, L., Schade, S., Sigurdsson, S., Steinitz, O., Stern, N., Teofilovski, A., Thorsson, J., Tomov, R., Tricarico, E., Trichkova, T., Tsiamis, K., Valkenburg, J. van, Vella, N., Verbrugge, L., Vétek, G., Villaverde, C., Witzell, J., Zenetos, A.,
Cardoso, A.C., 2018. Increasing understanding of alien species through citizen science (Alien-CSI). Res. Ideas Outcomes 4, e31412.

DOI: $10.3897 /$ rio.4.e31412

Vaissi, S., Sharifi, M., 2019. Integrating multi-criteria decision analysis with a GIS-based siting procedure to select a protected area for the Kaiser's mountain newt, Neurergus kaiseri (Caudata: Salamandridae). Glob. Ecol. Conserv. 20, e00738.

DOI: $\underline{10.1016 / \text { j.gecco.2019.e00738 }}$ 